\title{
ROWBOTTOM-TYPE PROPERTIES AND A CARDINAL ARITHMETIC
}

\author{
JAN TRYBA
}

\begin{abstract}
Assuming Rowbottom-type properties, we estimate the size of certain families of closed disjoint functions. We show that whenever $\kappa$ is Rowbottom and $2^{\omega}<\aleph_{\omega_{1}}(\kappa)$, then $2^{<\kappa}=2^{\omega}$ or $\kappa$ is the strong limit cardinal. Next we notice that every strongly inaccessible Jónsson cardinal $\kappa$ is $\nu$-Rowbottom for some $\nu<\kappa$. In turn, Shelah's method allows us to construct a Jónsson model of cardinality $\kappa^{+}$provided $\kappa^{\mathrm{cf}(\kappa)}=\kappa^{+}$. We include some additional remarks.
\end{abstract}

0. Introduction. In this paper the basic set-theoretical notation is standard. We only mention that the letters $\kappa, \lambda, \mu, \ldots$ are reserved for cardinals. All undefined notions are taken from [3].

By $j: M \rightarrow V_{\gamma}$ we mean an elementary embedding of the transitive model $M$ into the collection of all sets of rank less than the limit ordinal $\gamma$ which moves some ordinal. We write $(\kappa, \lambda) \rightarrow(\mu,<\nu)$ iff whenever $f:[\kappa]^{<\omega} \rightarrow \lambda$, there exists a set $X \subseteq \kappa$ such that $|X|=\mu$ and $\left|f^{\prime \prime}[X]^{<\omega}\right|<\nu$. A cardinal $\kappa>\nu$ is $\nu$-Rowbottom iff $(\kappa, \lambda) \rightarrow(\kappa,<\nu)$ for every $\lambda<\kappa ; \kappa$ is Rowbottom just in case it is $\omega_{1}$-Rowbottom. If $(\kappa, \nu) \rightarrow(\kappa,<\nu)$ for some $\nu<\kappa$, then $\kappa$ is called Jónsson.

Background information about a relation between Jónsson cardinals and elementary embeddings can be found in [8]. Mimicking [8] we can in fact prove that the property $(\kappa, \lambda) \rightarrow(\mu,<\nu)$ is equivalent to the existence of an elementary embedding $j: M \rightarrow V_{\gamma}$ such that $j(\delta)=\lambda$ for some $\delta<\nu, j(\tau)=\kappa$ for some $\tau \geq \mu$ and $\mu, \nu \in j^{\prime \prime} M$ for every $\gamma>\kappa$ (or equivalently, for some $\gamma>\kappa$; we may additionally demand that the model $V_{\gamma}$ carries countably many relations and operations).

1. Closed disjoint functions. We say that two functions $f$ and $g$ on $\lambda$ are closed disjoint iff the set $\{\alpha<\lambda: f(\alpha) \neq g(\alpha)\}$ contains a closed unbounded subset of $\lambda$.

Proposition 1.1. Assume $j: M \rightarrow V_{\gamma}, j(\delta)=\lambda$ for some $\delta<\lambda, \lambda$ is regular and $\mu \in M \cap j^{\prime \prime} M$. Let $h: \lambda \rightarrow \mu$. Then every family of closed disjoint functions $f \in \prod_{\alpha<\lambda} h(\alpha)$ has less than $j(\mu)$ elements.

ProOF. Suppose the opposite: there exist a function $h: \lambda \rightarrow \mu$ and a family of size $j(\mu)$ of closed disjoint functions $f \in \prod_{\alpha<\lambda} h(\alpha)$. Let $j(\tau)=\mu$. By elementarity and absoluteness we find in $M$ a function $h: \delta \rightarrow \tau$ and a family $F \in M$ of size $\mu$ of closed disjoint-in- $M$ functions $f \in \prod_{\alpha<\delta} \bar{h}(\alpha)$. Put $\eta=\sup j^{\prime \prime} \delta<\lambda$.

We shall prove that $(j(f))(\eta) \neq(j(g))(\eta)$ for any distinct $f, g \in F$. Let $C \subseteq$ $\{\alpha<\delta: f(\alpha) \neq g(\alpha)\}$ be closed unbounded in $\delta$ and $C \in M$. Therefore $j^{\prime \prime} C$ is

Received by the editors October 26, 1984.

1980 Mathematics Subject Classification. Primary 03E10, 03E55; Secondary 03C50, $03 E 05$.

Key words and phrases. Closed disjoint functions, $I$-functions, norm of a function, Chang's Conjecture, $\nu$-Rowbottom cardinals, Jónsson cardinals, possible scale for a sequence of ordinals. 
unbounded in $\sup j^{\prime \prime} \delta=\eta, j(C) \subseteq\{\alpha<\lambda:(j(f))(\alpha) \neq(j(g))(\alpha)\}$ and $j(C)$ is closed in $\lambda$. Since $j^{\prime \prime} C \in j(C)$, we have $\eta \in j(C)$.

Now we derive a contradiction, because

$$
|F|=|\{(j(f))(\eta): f \in F\}| \leq(j(\bar{h}))(\eta)<\mu .
$$

In the presence of Proposition 1.1 we receive some generalizations of the known results about Chang's Conjecture or those formulated in [7], for instance.

COROLlARY 1.2. If $\lambda$ is a regular cardinal and $(\kappa, \lambda) \rightarrow(\mu,<\lambda)$, then every family of closed disjoint functions $f: \lambda \rightarrow \eta$, where $\eta<\mu$, has less than $\kappa$ members. If in addition $\operatorname{cf}(\mu)>\lambda$, then every family of closed disjoint functions $f: \lambda \rightarrow \mu$ has at most $\kappa$ elements.

In particular, if $\kappa$ is Rowbottom, then the above statement is true for each regular $\omega<\lambda<\kappa=\mu$.

Let $I$ be an ideal over $\lambda$. A partial function $f$ on $\lambda$ is called an $I$-function iff $\operatorname{dom}(f) \notin I$ (compare [3, p. 432]). Two $I$-functions $f$ and $g$ on $\lambda$ are almost disjoint iff the set $\{\alpha<\lambda: f(\alpha)=g(\alpha)\}$ has size less than $\lambda$.

The method of the proof of Proposition 1.1 yields

Proposition 1.3. Assume $j: M \rightarrow V_{\gamma}, j(\delta)=\lambda$ for some $\delta<\lambda, \lambda$ is regular, $\lambda \leq \mu \in j^{\prime \prime} M$ and $\mu^{+} \in M$. Let I be any ideal over $\lambda$ containing all bounded subsets of $\lambda$. Then every family of almost disjoint I-functions on $\lambda$ into $\mu$ has less than $j\left(\mu^{+}\right)$elements.

PROOF. Argue by contradiction. Let $j(\tau)=\mu$. By our assumption there exist an $M$-ideal $I \in M$ over $\delta$ containing all bounded subsets of $\delta$ and a family $F \in M$ of size $\mu^{+}$of almost disjoint $\bar{I}$-functions on $\delta$ into $\tau$. Set $\eta=\sup j^{\prime \prime} \delta<\lambda$.

For any distinct $f, g \in F$ we can choose $\beta<\delta$ such that $f(\alpha) \neq g(\alpha)$ for all $\alpha \in \operatorname{dom}(f) \cap \operatorname{dom}(g), \alpha \geq \beta$. Thus $(j(f))(\alpha) \neq(j(g))(\alpha)$ for all $\alpha \in \operatorname{dom}(j(f)) \cap$ $\operatorname{dom}(j(g)), \alpha \geq \eta>j(\beta)$.

Since all bounded subsets of $\lambda$ are elements of $j(I)$, there is a subfamily $G \subseteq F$ of size $\mu^{+}$and an ordinal $\eta \leq \alpha<\lambda$ such that $\alpha \in \operatorname{dom}(j(f))$ for all $f \in G$. However, $|G|=|\{(j(f))(\alpha): f \in G\}| \leq \mu$, which is false.

COROLLARY 1.4. If $j: M \rightarrow V_{\gamma}, j(\delta)=\lambda$ for some $\delta<\lambda, \lambda$ is regular and $\lambda^{+} \in M$, then the ideal $I=\{x \subseteq \lambda:|x|<\lambda\}$ of bounded subsets of $\lambda$ is $j\left(\lambda^{+}\right)$saturated. Hence, the ideal I is $\lambda^{++}$-saturated, assuming $\left(\lambda^{++}, \lambda\right) \rightarrow\left(\lambda^{+},<\lambda\right)$.

PROOF. If $|x|=\lambda$, then the identity restricted to $x$ is an I-function. Now apply Proposition 1.3.

If $f$ and $g$ are ordinal-valued functions on a regular cardinal $\lambda>\omega$, then the symbol $g<f$ means that the set $\{\alpha<\lambda: g(\alpha)<f(\alpha)\}$ contains some closed unbounded subset of $\lambda$ (compare [3, p. 67]). The relation $g<f$ is well-founded and the rank $\|f\|=\sup \{\|g\|+1: g<f\}$ of $f$ in this one is called the norm of $f$. $\|\tau\|$ denotes the norm of the constant function on $\lambda$ taking $\tau$ as the only value.

The following result can be deduced indirectly from Shelah's work [5] using Magidor's filters. 
PROPOSITION 1.5. If $j: M \rightarrow V_{\gamma}, j(\delta)=\lambda$ for some $\delta<\lambda$ and $\lambda$ is regular, then $\|\tau\| \leq j(\tau)$ for each $\tau \in M \cap j^{\prime \prime} M$.

ProOF. Let $\eta=\sup j^{\prime \prime} \delta<\lambda$. First of all we want to show that $\|f\|^{M} \leq$ $(j(f))(\eta)$ for every ordinal-valued function $f \in M$ defined on $\delta$. This is done by simple induction on the norm $\|f\|^{M}$. If $\|f\|^{M}=\beta+1$, then there is some function $g \in M$ on $\delta$ such that $g<f$ and $\|g\|^{M}=\beta \leq(j(g))(\eta)$. But the proof of Proposition 1.1 shows that $g<f$ implies $(j(g))(\eta)<(j(f))(\eta)$ and so $\|f\|^{M}=\beta+1 \leq(j(f))(\eta)$. The case of limit $\|f\|^{M}$ is similar.

Presently, if $\|\tau\|>j(\tau)$ and $j(\alpha)=\tau$, then there exists a function $g: \lambda \rightarrow \tau$ such that $\|g\|=j(\tau)$ and so in $M$ there is a function $f: \delta \rightarrow \alpha$ such that $\|f\|^{M}=\tau$. But then $\tau \leq(j(f))(\eta)<j(\alpha)=\tau$, a contradiction.

COROLLARY 1.6. If $\lambda$ is a regular cardinal and $(\kappa, \lambda) \rightarrow(\mu,<\lambda)$, then $\|\mu\| \leq \kappa$. In particular, Chang's Conjecture $\left(\lambda^{+}, \lambda\right) \rightarrow(\lambda,,<\lambda)$ implies $\|\lambda\|=\lambda^{+}$.

2. Cardinal exponentiation. Using some elementary embeddings we can obtain a few inequalities in cardinal arithmetic.

LEMMA 2.1. If $j: M \rightarrow V_{\gamma}, j(\delta)=\lambda, \rho \in j^{\prime \prime} M, \mu=\left(\rho^{\delta}\right)^{+}$and $\mu \in M$, then $\rho^{\lambda}<j(\mu)$.

ProOF. Let $j(\eta)=\rho$ and assume to the contrary that $\rho^{\lambda} \geq j(\mu)$. Hence there exists some function from ${ }^{\lambda} \rho$ onto $j(\mu)$. So in $M$ there is a function which transforms $\left({ }^{\delta} \eta\right)^{M}$ onto $\mu$. As $\eta \leq \rho$, the contradiction $\mu \leq\left|\left({ }^{\delta} \eta\right)^{M}\right| \leq \rho^{\delta}<\mu$ establishes the Lemma.

COROLLARY 2.2. If $(\kappa, \lambda) \rightarrow(\mu,<\nu)$ and $\rho^{\alpha}<\mu$ for all $\alpha<\nu$, then $\rho^{\lambda}<\kappa$. Therefore, if $\kappa$ is $\nu$-Rowbottom and $2^{\alpha}<\kappa$ for all $\alpha<\nu$, then $\kappa$ is the strong limit cardinal.

REMARK 2.3. If $2^{\omega}<\aleph_{\omega}$ and $\aleph_{\omega}$ is Rowbottom, then $\aleph_{\omega}$ is the strong limit cardinal. By Theorem 84 from [3] and Corollary 1.4 we can even evaluate that $2^{\aleph_{n+1}}=2^{\aleph_{n}}$ or $2^{\aleph_{n+1}}<j\left(\aleph_{n+2}\right)$ for all $n<\omega$, whenever $j: M \rightarrow V_{\gamma}$ is an arbitrary elementary embedding such that $j\left(\aleph_{\omega}\right)=\aleph_{\omega}$ and some countable ordinal is moved by $j$.

REMARK 2.4. If a cardinal $\kappa$ is not strong limit, then the property $(\kappa, \lambda) \rightarrow$ $(\kappa,<\lambda)$ fails for the least $\lambda<\kappa$ such that $2^{\lambda} \geq \kappa$.

The same arguments can be used for proving

LEMMA 2.5. If $j: M \rightarrow V_{\gamma}, j(\delta)=\lambda, \rho \in j^{\prime \prime} M, \mu=\left(\delta^{\rho}\right)^{+}$and $\mu \in M$, then $\lambda^{\rho}<j(\mu)$.

COROLLARY 2.6. If $(\kappa, \lambda) \rightarrow(\mu,<\nu)$ and $\alpha^{\rho}<\mu$ for all $\alpha<\nu$, then $\lambda^{\rho}<\kappa$. Therefore, if $\kappa$ is $\nu$-Rowbottom and $\alpha^{\omega}<\kappa$ for all $\alpha<\nu$, then $\lambda^{\omega}<\kappa$ for all $\lambda<\kappa$.

LEMMA 2.7. If $(\kappa, \lambda) \rightarrow(\mu,<\lambda), \operatorname{cf}(\nu)=\lambda$ and $\alpha^{\lambda}<\mu$ for all $\alpha<\nu \leq \mu$, then $\nu^{\lambda}<\kappa$. Hence $2^{\nu}<\kappa$, if $\nu \leq \mu$ is a strong limit singular cardinal with $\operatorname{cf}(\nu)=\lambda$.

PROOF. Choose an elementary embedding $j: M \rightarrow V_{\kappa+\omega}$ such that $j(\delta)=\lambda$ for some $\delta<\lambda, j(\tau)=\kappa$ for some $\tau \geq \mu$ and $\mu, \nu \in j^{\prime \prime} M$. But $j(\nu)=\nu$ implies

$$
\lambda=\operatorname{cf}(j(\nu))=j\left(\mathrm{cf}^{M}(\nu)\right) \geq j(\lambda)>j(\delta)=\lambda .
$$


Thus $\nu$ is moved by $j$ and so $j$ witnesses $(\kappa, \nu) \rightarrow(\mu,<\nu)$. Now Corollary 2.6 completes the proof.

Galvin-Hajnal's method [2] and Corollary 1.6 allow us to formulate some bounds on $\nu^{\mathrm{cf}(\nu)}$ in certain cases of singular cardinals $\nu>\mu$.

THEOREM 2.8. Assume $(\kappa, \lambda) \rightarrow(\mu,<\lambda)$. Let $\lambda \leq \eta \leq \mu$ and $\nu=\aleph_{\eta}$. If $\operatorname{cf}(\eta)=\lambda$ and $\alpha^{\lambda}<\nu$ for all $\alpha<\nu$, then $\nu^{\lambda}<\aleph_{\kappa}$. In particular, if $\nu$ is the strong limit singular cardinal of cofinality $\lambda$, then $2^{\nu}<\aleph_{\kappa}$.

Corollary 2.9 (Magidor [4]). Presuming Chang's Conjecture $\left(\lambda^{+}, \lambda\right) \rightarrow$ $(\lambda,<\lambda)$, if $\aleph_{\lambda}$ is the strong limit cardinal, then $2^{\aleph_{\lambda}}<\aleph_{\lambda^{+}}$.

COROLLARY 2.10. If $\aleph_{\omega}$ is Rowbottom and $\aleph_{\omega_{n}}$ is the strong limit cardinal for some $n<\omega$, then $2^{\aleph_{\omega_{n}}}<\aleph_{\omega_{\omega}}$.

The main theorem is based on the following technical

CLAIM 2.11. Let $\lambda$ be the least cardinal such that $\rho^{\lambda}>\rho^{\nu}$. If $(\kappa, \lambda) \rightarrow(\mu,<\lambda)$, $\lambda<\mu, \operatorname{cf}(\mu) \neq \lambda$ and $\rho^{\nu}<\aleph_{\lambda}(\mu)$, then $\rho^{\lambda} \leq \kappa$. Moreover, $\rho^{\lambda}<\kappa$ unless $\kappa$ is singular.

ProOF. Simple arithmetic shows that $\lambda$ is regular. Clearly, $\nu<\lambda \leq \rho^{\nu}$. Assigning for each $f \in{ }^{\lambda} \rho$ the sequence $\bar{f}=\langle f \mid \alpha: \alpha<\lambda\rangle$ we get the branching family $F$ of $\rho^{\lambda}$ functions from $\lambda$ into some set of cardinality $\rho^{\nu}$ (whenever $\bar{f}, \bar{g} \in F$ and $\bar{f}(\beta)=\bar{g}(\beta)$, then $\bar{f}(\alpha)=\bar{g}(\alpha)$ for all $\alpha \leq \beta$-compare [3, p. 431]).

Let $\rho^{\nu}<\sigma \leq \rho^{\lambda}$ be any regular cardinal. As $\operatorname{cf}(\eta) \neq \lambda$ for all cardinals $\mu \leq \eta<$ $\aleph_{\lambda}(\mu)$, the proof of Lemma 35.2 in $[3]$ shows how then to construct a branching family $G$ of $\sigma$ functions $f: \lambda \rightarrow \eta$ for some $\eta<\mu, \eta \leq \lambda$ or $\operatorname{cf}(\eta)=\lambda$. But Corollary 1.2 gives $|G|<\kappa$, so we are done.

LEMMA 2.12. If $\nu<\mu, \operatorname{cf}(\mu) \leq \nu$ or $\mu$ is regular, $(\kappa, \lambda) \rightarrow(\mu,<\lambda)$ holds for every regular $\nu<\lambda<\mu$ and $\kappa^{-} \leq \rho^{\nu}<\aleph_{\nu^{+}}(\mu)$, then $\rho^{<\mu}=\rho^{\nu}$.

COROLlary 2.13. Assume $\left(\lambda^{++}, \lambda\right) \rightarrow\left(\lambda^{+},<\lambda\right)$. Then $2^{<\lambda}=\lambda$ implies $2^{\lambda}=\lambda^{+}$and $\lambda^{+} \leq 2^{<\lambda}<\aleph_{\lambda}$ implies $2^{\lambda}=2^{<\lambda}$.

THEOREM 2.14. Let $\nu=\sup \{\lambda<\kappa: \lambda$ is regular and $(\kappa, \lambda) \rightarrow(\kappa,<\lambda)$ fails $\}$. If $\nu<\kappa$ and $\kappa^{-} \leq 2^{\nu}<\aleph_{\nu^{+}}(\kappa)$, then $2^{<\kappa}=2^{\nu}$. In particular, if $\kappa$ is $\nu^{+}$. Rowbottom and $\kappa \leq 2^{\nu}<\aleph_{\nu^{+}}(\kappa)$, then $2^{<\kappa}=2^{\nu}$.

PROOF. If $\nu<\kappa$ then $(\kappa, \lambda) \rightarrow(\kappa,<\lambda)$ for every regular $\nu<\lambda<\kappa$. It is easy to see that then $\kappa$ is regular or $\operatorname{cf}(\kappa) \leq \nu$ (compare Lemma 2 in [8]). Applying Lemma 2.12 we finish the proof.

COROLLARY 2.15. If $\aleph_{\omega}$ is Rowbottom and $\aleph_{\omega}<2^{\aleph_{n}}<\aleph_{\omega_{n+1}}$ for some $n<\omega$, then $2^{\aleph_{\omega}}=2^{\aleph_{n}}$. $2^{<\nu}$.

REMARK 2.16. If $\kappa$ is $\nu$-Rowbottom and $\kappa \leq 2^{<\nu}<\aleph_{\mathrm{cf}(\nu)}(\kappa)$, then $2^{<\kappa}=$

QUESTION 2.17. Under the notation of Theorem 2.14, is $\nu<\kappa$ whenever $\kappa$ is Jónsson? 
3. The first critical point. The least ordinal moved by an elementary embedding $j: M \rightarrow V_{\gamma}$ is regular in $M$. Thus the correspondence between Rowbottomtype properties and elementary embeddings shows that the first cardinal $\nu \leq \mu$ such that $(\kappa, \nu) \rightarrow(\mu,<\nu)$ is regular. We shall prove that such $\nu$ cannot be strongly inaccessible, whenever $\mu=\kappa$.

LEMMA 3.1. Assume that $(\kappa, \nu) \rightarrow(\kappa,<\nu)$ and $\nu<\kappa$ is a limit cardinal. Let $\lambda \leq \kappa$ be the least cardinal such that $\lambda>\nu$ and the property $(\kappa, \lambda) \rightarrow(\kappa,<\lambda)$ fails. If $\nu^{\operatorname{cf}(\nu)}<\lambda$, then there exists $\rho<\nu$ such that $(\kappa, \nu) \rightarrow(\kappa,<\rho)$.

Proof. Set $A=\{\sigma<\nu:(\kappa, \sigma) \rightarrow(\kappa,<\sigma)$ fails $\}$ and $\eta=\sup A$. If $\eta<\nu$, then our statement is true for $\rho=\eta^{+}$. If this were false, there would be some strictly increasing sequence $\left\langle\sigma_{\alpha}: \alpha<\operatorname{cf}(\nu)\right\rangle$ of elements of $A$, cofinal in $\nu$. For each $\alpha<\operatorname{cf}(\nu)$ we can find a partition $f_{\alpha}:[\kappa]^{<\omega} \rightarrow \sigma_{\alpha}$ such that $\left|f_{\alpha}^{\prime \prime}[X]^{<\omega}\right|=\sigma_{\alpha}$ for every $X \subseteq \kappa$ of size $\kappa$ (a counterexample for $\left(\kappa, \sigma_{\alpha}\right) \rightarrow\left(\kappa,<\sigma_{\alpha}\right)$ ).

Put $B=\prod_{\alpha<\operatorname{cf}(\nu)} \sigma_{\alpha}$ and define $g:[\kappa]^{<\omega} \rightarrow B$ setting $g(s)=\left\langle f_{\alpha}(s): \alpha<\operatorname{cf}(\nu)\right\rangle$ for each finite subset $s \subseteq \kappa$. As $|B|=\nu^{\text {cf }(\nu)}<\lambda$, the definition of $\lambda$ supplies a set $X \subseteq \kappa$ such that $|X|=\kappa$ and $\left|g^{\prime \prime}[X]<\omega\right|<\nu$. On the other hand, $\left|g^{\prime \prime}[X]<\omega\right| \geq$ $\sup _{\alpha<\operatorname{cf}(\nu)}\left|f_{\alpha}^{\prime \prime}[X]<\omega\right| \geq \nu$ by definition of $g$. This contradiction establishes the Lemma.

COROLLARY 3.2. If $\nu<\kappa$ is a strong limit cardinal and $(\kappa, \nu) \rightarrow(\kappa,<\nu)$, then $(\kappa, \nu) \rightarrow(\kappa,<\rho)$ for some $\rho<\nu$. Thus the least cardinal $\lambda<\kappa$ such that $(\kappa, \lambda) \rightarrow(\kappa,<\lambda)$ cannot be strongly inaccessible.

Proof. Let $\lambda \leq \kappa$ be the least cardinal such that $\lambda>\nu$ and $(\kappa, \lambda) \rightarrow(\kappa,<\lambda)$ fails. Observe that $\lambda$ is $\nu$-Rowbottom. Since $2^{<\nu}=\nu$, the cardinal $\lambda$ is strong limit by Corollary 2.2. Now $\nu^{\operatorname{cf}(\nu)}=2^{\nu}<\lambda$ and our claim follows from Lemma 3.1.

QUESTION 3.3. Is the least $\lambda<\kappa$ such that $(\kappa, \lambda) \rightarrow(\kappa,<\lambda)$ is always a successor cardinal?

THEOREM 3.4. Every strongly inaccessible Jónsson cardinal $\kappa$ is $\rho$-Rowbottom for some $\rho<\kappa$.

PROOF. Let $\lambda<\kappa$ be any regular cardinal such that $(\kappa, \lambda) \rightarrow(\kappa,<\lambda)$. The set $S=\{\nu<\kappa: \operatorname{cf}(\nu)=\lambda$ and $\nu$ is strong limit $\}$ is stationary in $\kappa$. Lemma 2 from [8] shows that $(\kappa, \nu) \rightarrow(\kappa,<\nu)$ for every $\nu \in S$. By Corollary 3.2, for each $\nu \in S$ we can choose some $\rho_{\nu}<\nu$ so that $(\kappa, \nu) \rightarrow\left(\kappa,<\rho_{\nu}\right)$. By Fodor's Theorem there exist some fixed $\rho<\kappa$ and a stationary subset $T \subseteq S$ such that $(\kappa, \nu) \rightarrow(\kappa,<\rho)$ for each $\nu \in T$. This means that $\kappa$ is $\rho$-Rowbottom, since $T$ is unbounded in $\kappa$.

QUESTION 3.5. May we erase the word "strongly" from the above Theorem?

4. Jónsson models and successor cardinals. We showed in [8] that a successor cardinal $\kappa^{+}$is not Jónsson, whenever $\kappa$ is regular. Alternatively, $\kappa^{+}$is not Jónsson under $2^{\kappa}=\kappa^{+}[\mathbf{1}]$. Shelah's method from [6] enables us to weaken this presumption.

We say that a regular cardinal $\mu$ is a possible scale for the sequence $\left\langle\kappa_{i}: i<\lambda\right\rangle$ of cardinals iff there exists a sequence $\left\langle f_{\alpha}: \alpha<\mu\right\rangle$ of functions on $\lambda$ such that

(i) $f_{\alpha} \in \prod_{i<\lambda} \kappa_{i}$ for all $\alpha<\mu$,

(ii) $\left|\left\{i<\lambda: f_{\alpha}(i) \geq f_{\beta}(i)\right\}\right|<\lambda$ for all $\alpha<\beta<\mu$. (We shall write $f_{\alpha}<f_{\beta}$.)

(iii) For every $f \in \prod_{i<\lambda} \kappa_{i}$ there exists $\alpha<\mu$ such that $\mid\left\{i<\lambda\right.$ : $\left.f(i) \leq f_{\alpha}(i)\right\} \mid=$ $\lambda$ (compare [6]). 
LEMMA 4.1 (ShelaH [6]). Let $j: M \rightarrow V_{\gamma}$ and $N=j^{\prime \prime} M$. If $\mu$ is a possible sclae for the sequence $\left\langle\kappa_{i}: i<\lambda\right\rangle \in N$ of regular cardinals, $\lambda+1 \subseteq N$ and $j(\mu)=\mu$, then $\left|\left\{i<\lambda: j\left(\kappa_{i}\right)=\kappa_{i}\right\}\right|=\lambda$.

ProOF. Set $A=\left\{i<\lambda:\left|N \cap \kappa_{i}\right|<\kappa_{i}\right\}$ and assume to the contrary that $|\lambda \backslash A|<\lambda$. As $N$ is the elementary substructure of $V_{\gamma}$, some sequence $\left\langle f_{\alpha}: \alpha<\right.$ $\mu\rangle \in N$ exemplifies that $\mu$ is a possible scale for $\left\langle\kappa_{i}: i<\lambda\right\rangle$. For each $i \in A$ the subset $B_{i}=\left\{f_{\alpha}(i): \alpha \in N \cap \mu\right\}$ of $N \cap \kappa_{i}$ has cardinality less than $\kappa_{i}$, so we may choose $\sup B_{i}<f(i)<\kappa_{i}$ by regularity of $\kappa_{i}$. Accepting $f(i)=0$ for $i \in \lambda \backslash A$, we have $f_{\alpha}<f$ for every $\alpha \in N \cap \mu$. As the relation $<$ is transitive and the set $N \cap \mu$ is cofinal in $\mu, f_{\alpha}<f$ for every $\alpha<\mu$, which is impossible.

THEOREM 4.2. If $\kappa^{\mathrm{cf}(\kappa)}=\kappa^{+}$, then $\kappa^{+}$is not Jónsson.

ProOF. By our first remark in this item we may assume that $\lambda=\operatorname{cf}(\kappa)<\kappa$. Suppose that $\kappa^{+}$is Jónsson and pick an elementary embedding $j: M \rightarrow V_{\kappa^{+}+\omega}$ such that $j(\alpha)=\alpha$ for all $\alpha \leq \lambda, j(\nu)>\nu$ for some $\nu<\kappa$ and $j\left(\kappa^{+}\right)=\kappa^{+}$(see the proof of Theorem 1 in $[8]$ ). Then there exists some strictly increasing sequence $\left\langle\kappa_{i}: i<\lambda\right\rangle \in j^{\prime \prime} M$ of cardinals, cofinal in $\kappa$, with $\kappa_{0} \geq \nu$.

Cantor's diagonalization method shows that every family of $\kappa$ functions $f \in$ $\prod_{i<\lambda} \kappa_{i}^{++}$has an upper bound in the relation $<$. Since $\left|\prod_{i<\lambda} \kappa_{i}^{++}\right|=\kappa^{\lambda}=\kappa^{+}$, $\kappa^{+}$is the only possible scale for $\left\langle\kappa_{i}^{++}: i<\lambda\right\rangle$. Now, by Lemma 4.1, the set $A=$ $\left\{i<\lambda: j\left(\kappa_{i}^{++}\right)=\kappa_{i}^{++}\right\}$is unbounded in $\lambda$. But each $\kappa_{i}^{++}$, where $i \in A$, is Jónsson, contradicting [8].

COROLLARY 4.3 (SHELAH [6]). If $2^{\aleph_{\alpha}}=\aleph_{\alpha+\gamma+1}$ and $\operatorname{cf}(\gamma)<\aleph_{\alpha+1}$, then $2^{\aleph_{\alpha}}$ cannot be Jónsson.

With a slight modification, a similar argument can be used for

LEMMA 4.4. If $\kappa^{+}$is Jónsson, $\lambda=\operatorname{cf}(\kappa)>\omega$ and $\rho^{\lambda}<\kappa$ for $\rho<\kappa$, then the set $\left\{\rho<\kappa: \rho^{+}\right.$is Jónsson $\}$contains some closed unbounded subset of $\kappa$.

PROOF. Let $j: M \rightarrow V_{\kappa^{+}+\omega}, j(\alpha)=\alpha$ for all $\alpha \leq \lambda, j(\nu)>\nu$ for some $\lambda<\nu<\kappa$ and $j\left(\kappa^{+}\right)=\kappa^{+}$. Choose a strictly increasing continuous sequence $\left\langle\rho_{i}: i<\lambda\right\rangle \in j^{\prime \prime} M$ of cardinals, cofinal in $\kappa$, with $\rho_{0} \geq \nu$. Suppose by way of contradiction that the set $S=\left\{i<\lambda: \rho_{i}^{+}\right.$is not Jónsson $\}$is stationary in $\lambda$.

Set $\kappa_{i}=\rho_{i}^{+}$for $i \in S$ and $\kappa_{i}=\rho_{i}^{++}$for $i \in \lambda \backslash S$. Thus no $\kappa_{i}$ is Jónsson. Since $S$ is stationary, by an analogue of Lemma 8.5 stated in [3], every family of almost disjoint functions $f \in \prod_{i<\lambda} \kappa_{i}$ has at most $\kappa^{+}$elements. But every family of $\kappa$ functions $f \in \prod_{i<\lambda} \kappa_{i}$ has an upper bound in the relation $<$. Thus $\kappa^{+}$is the only possible scale for $\left\langle\kappa_{i}: i<\lambda\right\rangle$. Now each element of the set $\left\{\kappa_{i}: i<\lambda\right.$ and $\left.j\left(\kappa_{i}\right)=\kappa_{i}\right\} \neq \varnothing$ is Jónsson, contrary to our choice.

REMARK 4.5. We recall another result of Shelah from [6] which can be formulated as follows: If $\rho^{\operatorname{cf}(\kappa)}<\kappa$ for all $\rho<\kappa$ and $\kappa^{+}$is Jónsson, then the set $\{\lambda<\kappa: \lambda$ is a regular Jónsson cardinal $\}$ is unbounded in $\kappa$. Hence, if $\lambda$ is arbitrary and $\lambda^{\omega}=\aleph_{\alpha}$, then $\aleph_{\alpha+\omega+1}$ cannot be Jónsson.

We can also leave out one assumption in Shelah's result [6].

LEMMA 4.6. If $\left(\lambda^{+}\right)^{\omega}=\lambda^{+}$for all singular cardinals $\lambda$, then no successor cardinal is Jónsson. 
PROOF. Suppose to the contrary that $\kappa^{+}$is the least successor cardinal which is Jónsson. There are now two cases:

Case I: $\operatorname{cf}(\kappa)=\omega$. Then $\kappa^{\operatorname{cf}(\kappa)} \leq\left(\kappa^{+}\right)^{\omega}=\kappa^{+}$and a contradiction follows from Theorem 4.2.

Case II: $\kappa>\operatorname{cf}(\kappa)>\omega$. First, collapse $\operatorname{cf}(\kappa)$ onto $\omega_{1}$ using the notion of forcing $P=\left\{p: p\right.$ is a function with $\operatorname{dom}(p) \in \omega_{1}$ and $\left.\operatorname{ran}(p) \subseteq \operatorname{cf}(\kappa)\right\}$ ordered by inclusion.

Let $G$ be any generic filter on $P$. Since $|P|=\operatorname{cf}(\kappa)^{\omega}<\kappa$, it follows from [8] that $\kappa^{+}$remains Jónsson in the forcing extension $V[G]$ of a ground model $V$. Clearly, since $P$ is $\omega_{1}$-closed, $\omega_{1}$ is preserved and $\operatorname{cf}(\kappa)=\omega_{1}$ in $V[G]$. Moreover, $|P|$ is collapsed onto $\omega_{1}$ and every cardinal $\lambda>|P|$ in $V$ remains a cardinal in $V[G]$. The equality $\left(\lambda^{+}\right)^{\omega}=\lambda^{+}$is also true in $V[G]$ for every singular cardinal $\lambda$.

From now on work in $V[G]$. Let $j: M \rightarrow V_{\gamma}$ witness that $\kappa^{+}$is Jónsson. Pick some strictly increasing continuous sequence $\left\langle\kappa_{i}: i<\omega_{1}\right\rangle \in j^{\prime \prime} M$ of cardinals with cofinality $\omega$, cofinal in $\kappa$. Since $\left|\prod_{i<1} \kappa_{i}^{+}\right| \leq\left(\kappa_{1}^{+}\right)^{\omega}=\kappa_{1}^{+}$for all $1<\omega_{1}$, by Claim 13 from [6] the successor $\kappa^{+}$is a possible scale for $\left\langle\kappa_{i}^{+}: i<\omega_{1}\right\rangle$. It follows from Lemma 4.1 that the set $A=\left\{i<\omega_{1}: j\left(\kappa_{i}^{+}\right)=\kappa_{i}^{+}\right\}$is unbounded in $\omega_{1}$. But if $i \in A$ and $\kappa_{i}^{+}$is greater than the first ordinal moved by $j$, then $\kappa_{i}^{+}$is Jónsson, which is a contradiction because $\operatorname{cf}\left(\kappa_{i}\right)=\omega$ and $\left(\kappa_{i}\right)^{\omega}=\kappa_{i}^{+}$.

The same proof shows

Lemma 4.7. Assume the Singular Cardinals Hypothesis. Then $2^{\omega}<\kappa$ implies that $\kappa^{+}$is not Jónsson.

LEMMA 4.8. No successor cardinal above a compact cardinal is Jónsson.

PROOF. Solovay showed that the singular cardinals hypothesis holds above the least compact cardinal (see [3, p. 405]). Now proceed as in the proof of Lemma 4.6.

\section{REFERENCES}

1. P. Erdös and A. Hajnal, On a problem of B. Jónsson, Bull. Acad. Polon. Sci. Sér. Sci. Math. Astronom. Phys. 14 (1966), 19-23.

2. F. Galvin and A. Hajnal, Inequalities for cardinal powers, Ann. of Math. (2) 101 (1975), 491-498.

3. T. Jech, Set theory, Academic Press, New York, 1978.

4. M. Magidor, Chang's conjecture and powers of singular cardinals, J. Symbolic Logic 42 (1977), 272-276.

5. S. Shelah, A note on cardinal exponentiation, J. Symbolic Logic 45 (1980), 56-66.

6. __ Jónsson algebras in successor cardinals, Israel J. Math. 30 (1978), 57-64.

7. J. Tryba, A few remarks on Rowbottom cardinals, Israel J. Math. 40 (1981), 193-196.

8. __ On Jónsson cardinals with uncountable cofinality, Israel J. Math. 49 (1984), 315324.

Department of Mathematics, Gdańsk University, Wita Stwosza 57, 80-952 GDASŃK, POLAND 